\title{
Mapping and validation of major quantitative trait loci for kernel length in wild barley (Hordeum vulgare ssp. spontaneum)
}

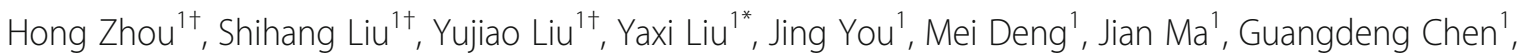 \\ Yuming Wei', Chunji Liu ${ }^{2}$ and Youliang Zheng ${ }^{1}$
}

\begin{abstract}
Background: Kernel length is an important target trait in barley (Hordeum vulgare L.) breeding programs. However, the number of known quantitative trait loci (QTLs) controlling kernel length is limited. In the present study, we aimed to identify major QTLs for kernel length, as well as putative candidate genes that might influence kernel length in wild barley.

Results: A recombinant inbred line (RIL) population derived from the barley cultivar Baudin (H. vulgare ssp. vulgare) and the long-kernel wild barley genotype Awcs276 (H.vulgare ssp. spontaneum) was evaluated at one location over three years. A high-density genetic linkage map was constructed using 1,832 genome-wide diversity array technology (DArT) markers, spanning a total of $927.07 \mathrm{cM}$ with an average interval of approximately $0.49 \mathrm{cM}$. Two major QTLs for kernel length, LEN-3H and LEN-4H, were detected across environments and further validated in a second RIL population derived from Fleet (H. vulgare ssp. vulgare) and Awcs276. In addition, a systematic search of public databases identified four candidate genes and four categories of proteins related to LEN-3H and LEN-4H.

Conclusions: This study establishes a fundamental research platform for genomic studies and marker-assisted selection, since $L E N-3 H$ and $L E N-4 H$ could be used for accelerating progress in barley breeding programs that aim to improve kernel length.
\end{abstract}

Keywords: Barley, Genetic linkage map, Kernel length, QTL, Validation, Candidate gene

\section{Background}

Barley (Hordeum vulgare L.) is one of the seven cereal crops grown worldwide and widely used in the animal feed and food industry. In 2012, barley was cultivated on 51.05 million hectares worldwide, resulting in the production of approximately 129.9 million metric tons (http:// www.fao.org/home/en/). Barley is diploid $(2 \mathrm{n}=14)$, and its seven chromosomes share homology with those of other cereal species such as wheat, rye, and rice; therefore,

\footnotetext{
*Correspondence: yaxi.liu@outlook.com; liuyaxi@sicau.edu.cn

${ }^{\dagger}$ Equal contributors

${ }^{1}$ Triticeae Research Institute, Sichuan Agricultural University, Wenjiang, Chengdu 611130, China

Full list of author information is available at the end of the article
}

it is an ideal species for genetic mapping and quantitative trait locus (QTL) analysis [1].

Significant progress has been made since the advent of molecular markers in genetic and QTL mapping. The first genetic map in barley was constructed using restriction fragment length polymorphism (RFLP) markers [2], whereas additional markers were used to build and improve barley linkage maps, including single nucleotide polymorphisms (SNPs), diversity array technology (DArT) markers, simple sequence repeats (SSRs), amplified fragment length polymorphisms (AFLPs), and sequencetagged sites (STSs) [3-6]. Linkage maps enable general scientific discoveries, such as genome organization, QTL detection, and synteny establishment, whereas high- 
density maps are a useful tool in crop improvement programs to identify molecular markers linked to QTLs.

In barley, kernel length (LEN) is a major breeding target, since it is significantly correlated with grain yield. In previous studies, multiple QTLs for LEN have been fine-mapped. Ayoub et al. [7] reported a QTL for LEN in chromosome (Chr.) 3H; Backes et al. [8] reported two QTLs for LEN in Chr. 4H and 7H; Walker [9] detected QTLs for endosperm hardness, grain density, grain size, and malting quality using rapid phenotyping tools, and reported that 11 QTLs associated with LEN were significantly correlated with endosperm hardness, but not with grain density, using digital image analysis. Major QTLs for LEN have been also identified in rice, soybean [10], and wheat [11]. In rice, several loci associated with seed size and grain yield, including GS3 [12], GL7/GW7 [13], qSW5/GW5 [14], TGW6 [15], An-1 [16], BG2 [17], OsSIZ1 [18], and DST [19], have been cloned through map-based cloning techniques. Of these, $A n-1$ encodes a bHLH protein and regulates awn development, kernel size, and kernel number [16]; BG2 regulates kernelrelated traits, including kernel thickness, kernel width, and thousand kernel weight [17]; OsSIZ1 encodes E3 ubiquitin-protein ligases and regulates the vegetative growth and reproductive development [18]; and DST is a zinc finger transcription factor that regulates the expression of Gnla/OsCKX2 and improves grain yield [19].

In the present study, a recombinant inbred line (RIL) population derived from a cross between the barley cultivar Baudin (H. vulgare ssp. vulgare) and its wild relative Awcs276 (H.vulgare ssp. spontaneum) was evaluated in one location over three years in order to: (a) construct a high-density genetic linkage map using 1,832 DArT markers; (b) identify QTLs for LEN; (c) validate major QTLs for LEN in a second RIL population derived from a cross between Fleet ( $H$. vulgare ssp. vulgare) and Awcs276; and (d) identify putative candidate genes that may influence LEN. Although many loci/QTLs for LEN have been identified previously in barley using markerassisted selection, the discovery of additional loci/QTLs is necessary to enhance our understanding of the intricate genetic basis of kernel morphology and phenotype variance. These findings will provide new insights to improve barley yield in breeding programs.

\section{Methods}

\section{RIL populations and phenotyping}

The spring barley cultivars Baudin and Fleet $(H$. vulgare ssp. vulgare) along with their wild relative Awcs276 ( $H$. vulgare ssp. spontaneum) were obtained from a collection assembled at the University of Tasmania and used to generate two RIL populations (Fig. 1) as described by Chen [20]. Awcs276, a long-kernel wild barley genotype from the Middle East, was used as the common parent

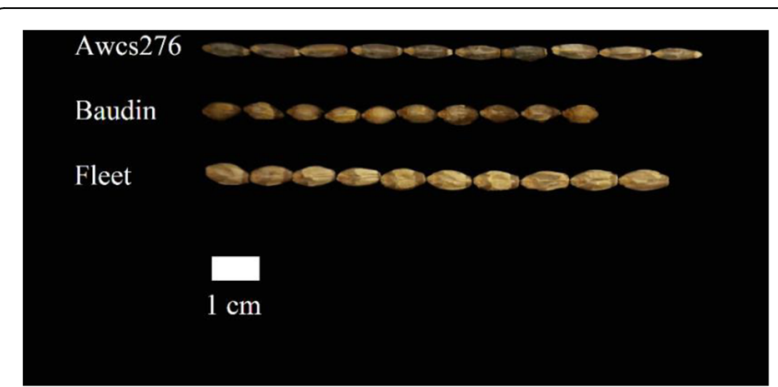

Fig. 1 Kernel phenotypes of Awcs276, Baudin, and Fleet used for quantitative trait locus mapping in this study. Kernels in the upper line belong to the long-kernel parent Awcs276, those in the lower line belong to the short-kernel parent Fleet, and those in the middle line belong to the short-kernel parent Baudin

in the two RIL populations (Baudin/Awcs276 and Fleet/ Awcs276). Baudin/Awcs276 (mapping population, 128 lines of $F_{8}, F_{9}$, and $F_{10}$ generations) was evaluated in one location over three years to detect QTLs for LEN, whereas Fleet/Awcs276 (validation population, 94 lines of $F_{10}$ generation) was evaluated for one year to validate putative QTLs identified in the mapping population. Baudin/Awcs276 was planted in October $2012\left(\mathrm{~F}_{8}\right), 2013$ $\left(F_{9}\right)$, and $2014\left(F_{10}\right)$ in duplicate rows of ten plants each in a completely randomized design in Wenjiang, Chengdu, China $\left(30^{\circ} 36^{\prime} \mathrm{N}, 103^{\circ} 41^{\prime} \mathrm{E}\right)$. The length of each row was $1.5 \mathrm{~m}$ with a row-to-row distance of $15 \mathrm{~cm}$. Field management was carried out according to common practices in barley production. Mixed seeds were collected from mature plants in May 2013, 2014, and 2015, dried, and stored at $25{ }^{\circ} \mathrm{C}$ until analysis. Fleet/ Awcs276 was planted in October 2014 and harvested in May 2015. Fully filled grains were used for measuring LEN in June 2015. LEN was measured in millimeters using a ruler and estimated by one measurement of 10 randomly selected kernels in 2013 or the average of three measurements in 2014 and 2015. The average LEN of each year was used for QTL analysis.

\section{Phenotypic data analysis}

LEN in a given environment was determined as the arithmetic average of three biological replicates. Student's $t$-test $(P<0.05)$ was used to identify the differences in LEN between the parental lines. Summary statistics were performed using Excel 2010 (Microsoft Corp., Redmond, WA, USA), whereas analysis of variance (ANOVA) in conjunction with Student's $t$-test $(P<0.001)$ using the general linear model (GLM) in SPSS 17.0 (IBM SPSS, Chicago, IL, USA). Broad-sense heritability $\left(H^{2}\right)$ for each trait was estimated as $H^{2}=\sigma_{\mathrm{g}}^{2} /\left(\sigma_{\mathrm{g}}^{2}+\sigma_{\mathrm{ge}}^{2} / \mathrm{n}+\sigma_{\mathrm{e}}^{2} / \mathrm{nr}\right)$, where $\sigma_{\mathrm{g}}^{2}$ is the genetic variance, $\sigma_{\mathrm{ge}}^{2}$ is the genotype by environment $(\mathrm{G} \times \mathrm{E})$ variance, $\sigma_{\mathrm{e}}^{2}$ is the error, $\mathrm{n}$ is the number of environments, and $\mathrm{r}$ is the number of replicates [21]. The $\sigma_{\mathrm{g}}^{2}, \sigma_{\mathrm{ge}}^{2}$, and $\sigma_{\mathrm{e}}^{2}$ values were calculated using 
ANOVA $(P<0.001)$ in SAS 9.2 (SAS Institute Inc., Cary, NC, USA). The best linear unbiased prediction (BLUP) method was used to estimate the random effects of mixed models. Phenotypic BLUP was calculated using the BLUP procedure in SAS 9.2.

\section{Genotyping and construction of genetic linkage map}

Total genomic DNA (gDNA) was isolated and purified from fresh leaf tissue of one randomly selected plant in each $F_{8}$ line of Baudin/Awcs276 and $F_{10}$ line of Fleet/ Awcs276 using the modified cetyltrimethylammonium bromide (CTAB) method [22]. DArT sequencing was conducted by Triticarte Pty Ltd. (Canberra, Australia), selecting the corresponding predominantly active genes of a genome fraction through the use of a combination of restriction enzymes, which separate low copy sequences from the repetitive fraction of the genome (http://www.diversityarrays.com/dart-application-dartseq). DArT sequencing generates two data types: 1) scores for "presence/absence" (dominant) markers, known as SilicoDArT markers, as they are analogous to microarray DArT markers, but are extracted in silico from sequences obtained from genomic representations; and 2) SNPs within the available genomic fragments. DArT loci were named according to their clone identification numbers as provided by Triticarte (http://www.diversityarrays.com/dartapplication-dartseq-data-types). Polymorphic loci were selected from a total of 62,216 DArT markers after discarding those with a minor allele frequency of 0.4 , a missing value of more than $20 \%$, or a common position.

The linkage map was constructed using IciMapping 3.2/4.0 [23] and JointMap4 [24]. All unanchored markers were properly grouped using IciMapping 3.2/4.0 with an LOD threshold of 3 . The linkage analysis was conducted using JoinMap 4 (Kyazma, Wageningen, Netherlands) with a recombination frequency of 0.25 , and all markers were grouped in the seven chromosomes.

\section{QTL mapping}

Phenotypic data of each trait were the means of three biological replications in a single environment. The phenotypic BLUP was used to detect QTLs from the combined three-year data. QTL analysis for selected environments was performed through the interval mapping (IM) using MAPQTL6.0 (Kyazma, Wageningen, Netherlands) [25]. A test of 1,000 permutations was used to identify the LOD threshold that corresponds to a genome-wide false discovery rate of $5 \%(P<0.05)$. QTLs that were stable for a target trait across environments with clearly overlapping positions on the same chromosome were assumed to be the same. Stable QTLs that explained more than $10 \%$ of the phenotypic variance for the specific trait were considered major QTLs [26].
QTLNetwork 2 [27] was used to determine QTLs with additive effects at individual loci, epistatic interactions between two different loci, and interactions between QTLs and the environment $(\mathrm{QTL} \times \mathrm{E})$. The analysis was based on a mixed linear model (MLM) with $2 \mathrm{cM}$ walking speed and 2D genome scan, which maps epistatic QTLs with or without single-locus effects using 1,000 permutations in order to generate a threshold for the presence of QTLs and QTL $\times$ E interactions.

\section{Marker development and QTL validation}

Sequence information was obtained from the IPK Barley Blast Server (http://webblast.ipk-gatersleben.de/barley/ index.php), and single-base differences were identified by high-resolution melt (HRM) analysis [28]. Markers were designed using Beacon Designer 7.9 and evaluated by Oligo 6.0 [29]. The parameters for Primer Premier (Premier Biosoft International, Palo Alto, CA, USA) were as follows: inner product size of $60-100 \mathrm{bp}$, melting temperature of $55 \pm 5{ }^{\circ} \mathrm{C}$, primer length of $20 \pm 3 \mathrm{bp}$, and $3^{\prime}$-end stability to avoid self-complementarity and primer dimer formation.

To detect markers, amplification reactions were performed in a total volume of $10 \mu \mathrm{l}$, containing $100 \mathrm{ng}$ of template DNA, $5 \mu \mathrm{l}$ of SsoFast EvaGreen mixture, 5 pmol of each forward and reverse primer, and DNase/ RNase-free water up to the final value. PCR conditions were adjusted according to primer sets as follows: $4 \mathrm{~min}$ at $94{ }^{\circ} \mathrm{C}, 50$ cycles of $1 \mathrm{~s}$ at $94{ }^{\circ} \mathrm{C}$, and $30 \mathrm{~s}$ at $55^{\circ} \mathrm{C}$. This process is a precise warming of the amplicon DNA from approximately $65{ }^{\circ} \mathrm{C}$ to $95{ }^{\circ} \mathrm{C}$. At some point during this process, the melting temperature of the amplicon is reached, and the two strands of DNA separate or "melt" apart [28].

The homozygous lines of Fleet/Awcs276 were used to validate major QTLs using the developed markers. Based on marker profiles, individuals were grouped into two classes: genotypes with homozygous alleles from AwcS276 and genotypes with homozygous alleles from Fleet. Student's $t$-test $(P<0.05)$ was used to calculate the differences in LEN between these two classes of alleles and measure QTL effects within the validation population.

\section{Putative candidate gene identification}

To identify putative coding gene regions, flanking candidate loci, or trait-related gene products, we used the corresponding QTL marker contigs to blast search against the WGSMorex database at the IPK Barley Blast Server (http:// webblast.ipk-gatersleben.de/barley/index.php). We obtained QTL positions within the Morex reference map and putative trait-related proteins. According to the putative protein categories, most genes controlling kernel traits were identified in rice. The sequences of identified genes in rice were 
used to perform a BLASTN search against the barley database of the National Center for Biotechnology Information (NCBI, http://www.ncbi.nlm.nih.gov/) and the Phytozome website (https://phytozome.jgi.doe.gov/pz/portal.html) in order to identify homologous candidate genes in barley and other cereal crops.

\section{Results}

\section{Phenotypic evaluation}

The parental lines Awcs276 and Baudin showed significant differences in LEN $(P<0.05)$ (Fig. 1, Additional file 1). The LEN (range, 7.12-7.97 mm; mean, $7.62 \mathrm{~mm}$ ) of Awcs276 was higher than that of Baudin (range, 6.75-7.68 mm; mean, $7.28 \mathrm{~mm}$ ). The trait variance over the three years and the phenotypic variance among RILs were high as shown by summary statistics, including range, mean, standard deviation, and coefficient of variation (Additional files 1, 2 and 3). The average LEN of 2013 was $8.11 \mathrm{~mm}$ (confidence interval, 8.011-8.192 mm), of 2014 was $7.25 \mathrm{~mm}$ (confidence interval, 7.185-7.313 $\mathrm{mm}$ ), and of 2015 was $7.87 \mathrm{~mm}$ (confidence interval, 7.787-7.949 mm). The frequency of LEN and transgressive segregations were observed over the three years, indicating the presence of favorable alleles. The minimum LEN was $6.38 \mathrm{~mm}$ and the maximum $9.4 \mathrm{~mm}$. The broad-sense heritability of LEN was low in $2013\left(h^{2}=0.122\right)$, owing to the lack of biological replications, high in $2014\left(h^{2}=0.937, \mathrm{~F}=16.33\right.$, $P<0.0001)$ and $2015\left(h^{2}=0.870, \mathrm{~F}=7.42, P<0.0001\right)$, and moderate $\left(h^{2}=0.622, \mathrm{~F}=11.5, P<0.0001\right)$ over the three years, suggesting that genetic factors played an important role in the formation of LEN (Additional file 2). LEN showed normal or near-normal distribution with quantitative inheritance patterns suitable for QTL identification (Additional file 4).

\section{Genetic linkage map construction}

A total of 1832 polymorphic markers (Additional file 5) was selected and mapped on eleven linkage groups (LGs) (Table 1, Additional file 6). The map spanned a total of $927.07 \mathrm{cM}$ with an average marker distance of $0.49 \mathrm{cM}$. The results showed that Chr. $1 \mathrm{H}$ contained LG1 with a length of $133.31 \mathrm{cM}$, Chr. 2H contained LG2 and LG3 with a length of $261.6 \mathrm{cM}$, Chr. $3 \mathrm{H}$ contained LG4 and LG5 with a length of $116.05 \mathrm{cM}$, Chr. 4H contained LG6 with a length of $112.55 \mathrm{cM}$, Chr. 5H contained LG7 and LG8 with a length of $88.42 \mathrm{cM}$, Chr. $6 \mathrm{H}$ contained LG9 with a length of $93.21 \mathrm{cM}$, and Chr. 7H contained LG10 and LG 11 with a length of $121.92 \mathrm{cM}$. The largest LG was LG2, which contained 289 DArT markers, and the smallest was LG8, which contained only 87 markers. On average, each LG contained 166.5 DArT markers and each Chr. contained 261.7 DArT markers. The genetic distances of the 11 LGs ranged from $22.10 \mathrm{cM}$ (LG8) to $196.24 \mathrm{cM}$ (LG2), and the
Table 1 Basic information regarding the barley genetic map

\begin{tabular}{lllcc}
\hline Chr. & Linkage & Marker number & Map length (cM) & Marker interval (cM) \\
\hline 1H & LG1 & 188 & 133.31 & 0.71 \\
$2 H$ & LG2 & 289 & 196.24 & 0.68 \\
& LG3 & 109 & 65.36 & 0.60 \\
$3 H$ & LG4 & 187 & 68.15 & 0.36 \\
& LG5 & 135 & 47.90 & 0.35 \\
4H & LG6 & 165 & 112.55 & 0.68 \\
$5 H$ & LG7 & 129 & 66.32 & 0.51 \\
& LG8 & 87 & 22.10 & 0.25 \\
$6 H$ & LG9 & 230 & 93.21 & 0.41 \\
7H & LG10 & 163 & 74.77 & 0.46 \\
& LG11 & 150 & 47.15 & 0.31 \\
Total & 1832 & 927.07 & 0.49 \\
\hline
\end{tabular}

Chr chromosome, LG linkage group, CM centimorgan

average marker distance spanned from $0.25 \mathrm{cM}$ (LG8) to $0.71 \mathrm{cM}$ (LG1) (Table 1). Our genetic map was compared with other consensus maps [5] and the Morex reference map, and the results showed that the marker order had a satisfactory correspondence across the seven chromosomes.

\section{QTL analysis and validation}

Five significant QTLs were detected for LEN across the three environments (Table 2). The phenotypic variance explained by individual QTLs ranged from $10.4 \%$ $(15 L E N-2 H)$ to $29.1 \%(L E N-3 H)$. We used interval mapping for QTL analysis, and identified QTLs on all the chromosomes, except for $1 \mathrm{H}$ and $5 \mathrm{H}$ (Table 2). Two QTLs for LEN, $L E N-3 H$ and $L E N-4 H$, were detected in different environments (Figs. 2 and 3); $L E N-3 H$ was identified in 2013 and 2014 and explained 29.1 and $22.3 \%$ of the phenotypic variance, respectively, whereas $L E N-4 H$ was identified in different environments, having an LOD score of 3.17-5.06. Except for the two major QTLs, the rest three were environment-specific. Using BLUP, we identified four QTLs (15LEN-2H, LEN-3H, $L E N-4 H$, and $14 L E N-6 H$ ) from the combined three-year data, all of which had positions similar to QTLs associated with the non-combined data. However, no QTLs were detected on $7 \mathrm{H}$ from the combined data (Table 2). Among the five QTLs for LEN, $L E N-3 H$ had additive main effects (a), whereas its interaction with the environment was not significant, showing high heritability (Table 3), whereas the rest four QTLs did not have additive effects.

Based on the sequences of tightly linked DArT markers, we BLAST-searched against the Ensembl Barley database at the Ensembl Plants Blast Server (http:// plants.ensembl.org) and found that $L E N-3 H$ was located on Chr. 3HL, whereas $L E N-4 H$ on Chr. 4HL. Next, we 
Table 2 Quantitative trait loci (QTLS) for LEN identified in the Baudin/Awcs276 recombinant inbred line (RIL) population

\begin{tabular}{|c|c|c|c|c|c|c|c|c|}
\hline $\mathrm{QTL}^{\mathrm{a}}$ & Chr. & Linkage & Environment & Left Marker & Right Marker & Range (cM) & LOD & $\%$ Expl. \\
\hline \multirow[t]{2}{*}{$15 L E N-2 H$} & $2 \mathrm{H}$ & LG3 & $15 \mathrm{WJ}$ & $3254852|F| 0-65: C>A$ & $6270031|F| 0-48: C>G-48: C>G$ & $16.326-17.508$ & 3.11 & 10.4 \\
\hline & & & Combined & $3254852|F| 0-65: C>A$ & $6270031|F| 0-48: C>G-48: C>G$ & 16.326-17.508 & 3.35 & 11.2 \\
\hline \multirow[t]{3}{*}{ LEN-3H } & $3 \mathrm{H}$ & LG4 & 13WJ & 6255968 & $3258624|F| 0-41: C>A-41: C>A$ & $23.405-25.611$ & 5.07 & 29.1 \\
\hline & & & $14 \mathrm{WJ}$ & 3931871 & $3258624|F| 0-41: C>A-41: C>A$ & $20.731-25.611$ & 7.12 & 22.3 \\
\hline & & & Combined & 6249147 & $3258624|F| 0-41: C>A-41: C>A$ & $21.375-25.611$ & 6.02 & 19.2 \\
\hline \multirow[t]{3}{*}{ LEN-4H } & $4 \mathrm{H}$ & LG6 & $14 \mathrm{WJ}$ & $5249122|F| 0-25: G>A-25: G>A$ & $3263178|F| 0-25: C>A-25: C>A$ & $68.431-69.947$ & 3.17 & 10.6 \\
\hline & & & $15 \mathrm{WJ}$ & 3910814 & $5249122|F| 0-25: G>A-25: G>A$ & $62.983-68.431$ & 5.06 & 16.4 \\
\hline & & & Combined & 3396110 & 4007032|F|0-46:C > A-46:C > A & 59.535-69.392 & 5.31 & 17.2 \\
\hline \multirow[t]{2}{*}{ 14LEN-6H } & $6 \mathrm{H}$ & LG9 & $14 \mathrm{WJ}$ & $4594605|F| 0-25: A>G-25: A>G$ & 3259546|F|0-62:A > T-62:A > T & $56.031-59.463$ & 5.47 & 17.6 \\
\hline & & & Combined & $4594605|F| 0-25: A>G-25: A>G$ & 3259546|F|0-62:A > T-62:A > T & $56.031-59.463$ & 3.92 & 13 \\
\hline 14LEN-7H & $7 \mathrm{H}$ & LG11 & 14WJ & $3429688|F| 0-38: T>C$ & $3256863|F| 0-29: G>A-29: G>A$ & $19.095-22.504$ & 5.31 & 17.2 \\
\hline
\end{tabular}

Chr chromosome, LG linkage group, CM centimorgan, Combined combined data over the three years of study, \% Expl the percentage of variance explained by QTL ${ }^{a}$ QTLs were identified by Interval Mapping (IM) using MAPQTL6.0, and a test of 1,000 permutations was used to identify the LOD threshold, corresponding to a genome-wide false discovery rate of $5 \%(P<0.05)$

BLAST-searched the sequences of tightly linked DArT markers against the Morex reference map database and converted DArT markers to HRM markers for tracking QTLs using quantitative real-time PCR. Accordingly, two primer pairs were designed and used to track $L E N$ $3 H$ and $L E N-4 H$ (Additional file 7).

In this study, two major QTLs were validated in Fleet/ Awcs276 (Table 4). For LEN-3H, the average LEN of genotypes with homozygous alleles from Awcs276 was significantly higher $(P<0.05)$ than that of genotypes with homozygous alleles from Fleet. Similarly, for $L E N-4 H$, the average LEN of genotypes with homozygous alleles from Awcs276 was significantly higher $(P<0.05)$ than that of genotypes with homozygous alleles from Fleet. Detailed information is presented in Additional files 8 and 9.

\section{Putative candidate genes}

For the two major QTLs for LEN in Baudin/Awcs276, we found several putative candidate genes for kernelrelated traits, and these genes could be divided into four categories (Table 5): the first category included genes related to defense response such as salt tolerance; the second category included genes related to receptors such as ethylene receptors; the third category included genes related to transcription factors and promoters such as basic helix-loop-helix (bHLH) DNA-binding superfamily proteins and MADS-box transcription factors; and the fourth category included genes related to various enzymes such as zinc finger $\mathrm{CCCH}$ domaincontaining proteins, E3 ubiquitin-protein ligases, and cytochrome P450.

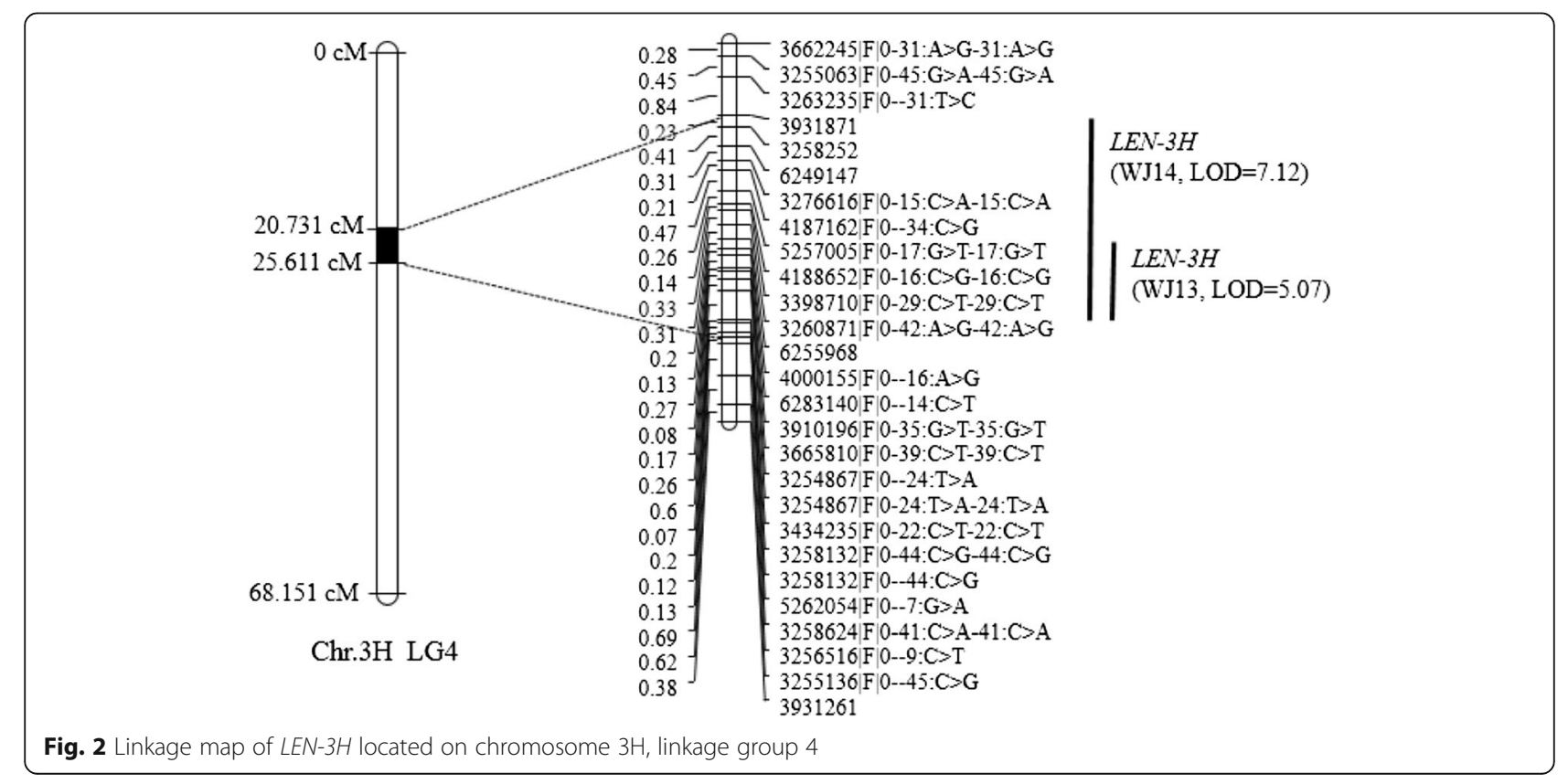




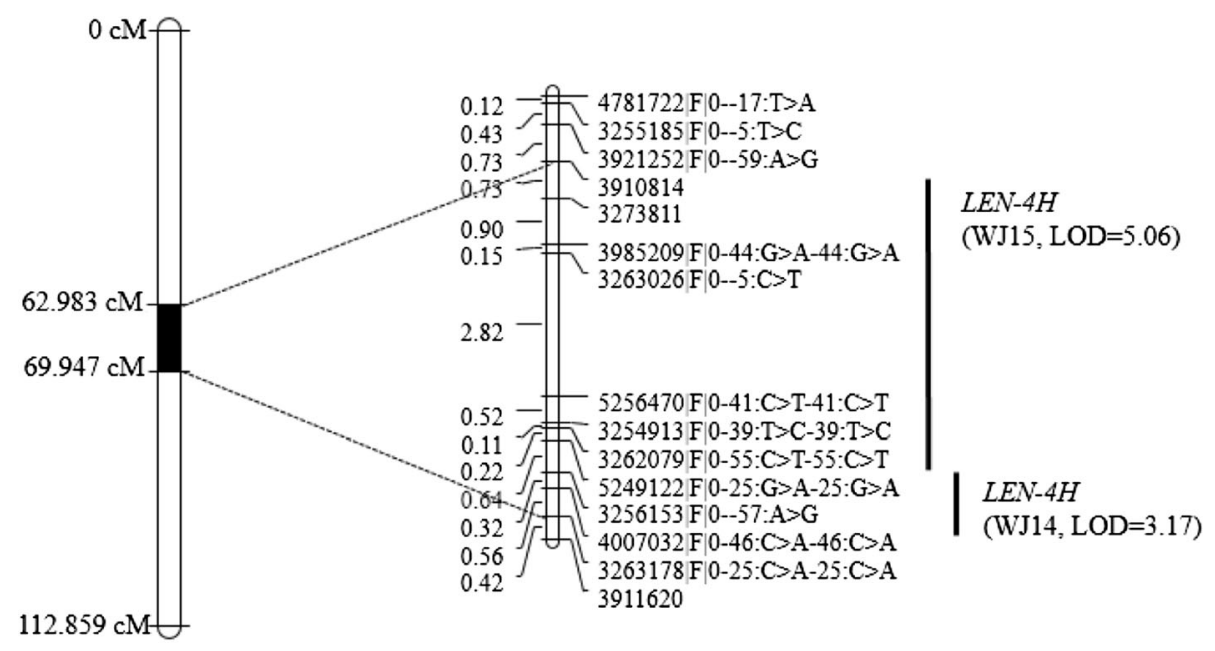

Chr.4H LG6

Fig. 3 Linkage map of LEN-4H located on chromosome 4 H, linkage group 6

\section{Discussion}

Awcs276 is a long-kernel wild barley genotype that has been previously used in genetic studies, because of its relatively long seeds, extensive environmental adaption, and high genetic diversity that can provide abundant germplasm resources for genetic variation and crop improvement [20,30,31]. Awcs276 was used in the present study owing to its having genes that are superior for LEN to those of the Australian barley cultivars Baudin and Fleet. Therefore, two RIL populations were developed by crossing Awcs276 with Baudin and Fleet to identify QTLs for LEN. Two major QTLs (LEN-3H and $L E N-4 H)$ were identified from Awcs276 in two environments. $L E N-3 H$ was detected in 2013 and 2014 in the interval of 20.731-25.611 cM on Chr. $3 \mathrm{H}$ using MAPQTL6.0. A peak within this interval was also identified in 2015 with a maximum LOD of 1.19, explaining $4.1 \%$ of the phenotypic variance (Additional file 10). Both the environmental variation and $\mathrm{G} \times \mathrm{E}$ interaction were highly significant $(P<0.0001)$ (Additional file 2$)$. These results showed that the environment influenced the QTLs, explaining the reason that none QTL was found in all the experimental years. The effects of $L E N-3 H$ and $L E N-4 H$ were evaluated in Fleet/Awcs276, and the results showed that these two QTLs stably increase LEN in barley.
A QTL for kernel length was identified between $55.8 \mathrm{cM}$ and $84.3 \mathrm{cM}$ on $\mathrm{Chr}$. $3 \mathrm{H}$ in a previous study [7]. Furthermore, five markers (ABG462, PSR156a, ABG453, ABG499, and M351316) were found within this interval, and information on the marker ABG453 was obtained from GrainGenes (http://wheat.pw.usda.gov/GG3/). Therefore, we used the parental lines and some extreme phenotypes in their progenies to confirm ABG453, and found that it was polymorphic for the parental lines. Backes et al. [8] reported a QTL for kernel length on Chr. $4 \mathrm{H}$ in an interval of $12 \mathrm{cM}$ and identified four markers (MWG2033, MWG0857, MWG0611, and MWG0921) within it. In the present study, we found the nearby loci of MWG2033 in the Hv-Consensus2006-Marcel-4H from GrainGenes and used the parental lines to confirm the nearby markers. The marker HVM40 was polymorphic for the parental lines with a distance of $4.1 \mathrm{cM}$ from MWG2033 in the consensus map. Thus, ABG453 and HVM40 were used for genotyping the lines of Baudin/Awcs276 (Additional file 11). Next, we used these two markers along with DArT markers to construct a genetic map and found that ABG453 (69.142 cM) and HVM40 (95.841 cM) were mapped on LG4 and LG6, respectively (Additional file 12). Using BLUP, we identified $L E N-3 H$ and $L E N-4 H$ in the interval of 20.428-25.917 cM and 59.02-69.119 cM,

Table 3 Estimated additive and additive x environmental interactions of QTLs for kernel length (LEN) in barley

\begin{tabular}{|c|c|c|c|c|c|c|c|c|c|c|c|}
\hline \multirow[t]{2}{*}{ QTL name } & \multirow{2}{*}{$\begin{array}{l}\text { Flanking } \\
\text { interval }\end{array}$} & \multirow[t]{2}{*}{ LOD } & \multirow[t]{2}{*}{ a effect ${ }^{a}$} & \multirow[t]{2}{*}{ ae1 } & \multirow[t]{2}{*}{ ae2 } & \multirow[t]{2}{*}{ ae3 } & \multicolumn{5}{|c|}{ QTL heritability } \\
\hline & & & & & & & $\mathrm{h}^{2}(\mathrm{a})$ & $h^{2}(a e)$ & $\mathrm{h}^{2}$ (ae1) & $\mathrm{h}^{2}(\mathrm{ae} 2)$ & $\overline{h^{2}(\mathrm{ae} 3)}$ \\
\hline LEN-3H & $23.4-25.6$ & 7.12 & $-0.1599^{*}$ & NS & NS & NS & 0.1217 & 0.0139 & 0.0056 & 0.0027 & 0.0129 \\
\hline
\end{tabular}

ae1, ae2, and ae3, QTL $\times$ environment interaction effect in 2013, 2014, and 2015, respectively

NS non-significant, ${ }^{*}$, significant at $P<0.001$

a The analysis was based on a mixed linear model (MLM) with 1,000 permutations

The mixed linear model (MLM) was used to calculate the estimated additive (a) and additive $\times$ environment interactions (ae) 
Table 4 Validation of two quantitative trait loci (QTLs) in the Fleet/Awcs276 recombinant inbred line (RIL) population

\begin{tabular}{lllll}
\hline QTL & Chr. & AA & BB & $P$ value \\
\hline LEN-3H & $3 \mathrm{H}$ & 8.79 & 9.05 & $0.01^{* *}$ \\
LEN-4H & $4 \mathrm{H}$ & 8.83 & 9.03 & $0.03^{*}$ \\
\hline
\end{tabular}

$A A$ homozygous alleles from Fleet, $B B$ homozygous alleles from Awcs276, Chr chromosome

${ }^{\text {a }}$ Student's t-test $(P<0.05)$ was used to identify differences between the parental lines; ${ }^{*}$, significant at $P<0.01 ;{ }^{*}$, significant at $P<0.05$

respectively. ABG453 (69.142 cM) and HVM40 (95.841 cM) were not included in the QTL interval, thus we speculated that the QTLs detected by Ayoub et al. [7] and Backes et al. [8] were not the same as $L E N-3 H$ and $L E N-4 H$. In general, the two QTLs for kernel size that were identified in this study were within a relatively small interval, which makes them an ideal target for breeding programs as well as for the characterization of gene(s) underlying this locus.

Kernel size is a major determinant of grain weight and an important yield component [32]. It refers to the space bounded by the husks, measured by LEN and width, and serves as a component of grain yield that determines kernel weight [33]. LEN was an important trait for barley domestication and has been a major target in barley breeding, because of its direct influence on grain yield. In the present study, according to four categories of putative proteins that influence LEN and several homologous candidate genes in Zea mays, Arabidopsis thaliana,
Brachypodium distachyon, Panicum hallii, and Sorghum bicolor, we identified four putative candidate genes (NCBI accession no. AK361814.1, AK365156.1, AK366345.1, and AK374135.1) (Table 5). The putative candidate gene (NCBI accession no. AK361814.1) for $L E N-4 H$ was homologous to $A n-1$ in rice. And $A n-1$ encodes a bHLH protein that positively regulates cell division, grain length, and awn elongation, but negatively regulates the grain number per panicle in rice [16]. The other three putative candidate genes (NCBI accession no. AK365156.1, AK366345.1, and AK374135.1) for $L E N-3 H$ were homologous to $D S T$, OsSIZ1, and BG2, respectively (Table 5). DST is a zinc finger transcription factor that improves grain yield and regulates the expression of Gnla/OsCKX2 [19]. Li et al. [34] reported that DSTreg1 enhances panicle branching and increases the grain number. And OsSIZ1 encodes E3 ubiquitin-protein ligases that regulate the growth and development in rice [18]. Wang et al. [35] reported that ossiz1 mutants have shorter primary and adventitious roots than wild-type plants, suggesting that OsSIZ1 is associated with the regulation of root architecture and acts as a regulator of the $\mathrm{Pi}(\mathrm{N})$ dependent responses in rice. BG2 encodes OsCYP78A13, which has a paralog in rice (Grain Length 3.2; GL3.2, LOC_Os03g30420) with distinct expression patterns [17]. CYP78A13 is highly expressed in seeds at 5-8 day after planting, whereas GL3.2 is specifically expressed in the roots [17]. Analysis of transgenic plants harboring either CYP78A13 or GL3.2 revealed that both genes can promote

Table 5 Putative genes or proteins of major quantitative loci (QTLs) for kernel length in barley

\begin{tabular}{|c|c|c|c|c|c|c|c|c|c|}
\hline $\begin{array}{l}\text { Stable } \\
\text { QTLs }\end{array}$ & Chr. & $\begin{array}{l}\text { Putative candidate } \\
\text { genes }\end{array}$ & $\begin{array}{l}\text { Gene in } \\
\text { rice }\end{array}$ & $\begin{array}{l}\text { Putative } \\
\text { genes in } \\
\text { barley }\end{array}$ & Zea mays & $\begin{array}{l}\text { Arabidopsis } \\
\text { thaliana }\end{array}$ & $\begin{array}{l}\text { Brachypodium } \\
\text { distachyon }\end{array}$ & $\begin{array}{l}\text { Panicum } \\
\text { hallii }\end{array}$ & Sorghum bicolor \\
\hline \multirow[t]{7}{*}{$L E N-3 H$} & \multirow[t]{7}{*}{$3 \mathrm{H}$} & $\begin{array}{l}\text { Zinc finger } \mathrm{CCCH} \\
\text { domain-containing } \\
\text { protein }\end{array}$ & DST & AK365156.1 & GRMZM2G089448 & AT4G33660 & Bradilg06420 & Pahal.101451 & Sobic.001G065500 \\
\hline & & $\begin{array}{l}\text { E3 ubiquitin-protein } \\
\text { ligase BRE1-like protein }\end{array}$ & OsSIZ1 & AK366345.1 & GRMZM2G155123 & AT5G60410 & Bradi2g38030 & Pahal.C01170 & Sobic.009G026500 \\
\hline & & Cytochrome P450 & $\begin{array}{l}\text { GE; CYP78A13; } \\
\text { BG2 }\end{array}$ & AK374135.1 & GRMZM2G138008 & AT1G74110 & Bradi4g35890 & Pahal.B03875 & Sobic.002G367600 \\
\hline & & \multicolumn{8}{|c|}{ Polyglutamine-binding protein 1} \\
\hline & & \multicolumn{8}{|l|}{ Ankyrin-repeat protein } \\
\hline & & \multicolumn{8}{|l|}{ FeS assembly protein } \\
\hline & & \multicolumn{8}{|c|}{ Calcium-dependent protein kinase } \\
\hline \multirow[t]{6}{*}{ LEN-4H } & $4 \mathrm{H}$ & $\begin{array}{l}\text { Basic helix-loop-helix } \\
\text { (bHLH) DNA-binding } \\
\text { Superfamily protein }\end{array}$ & $A n-1$ & AK361814.1 & GRMZM5G828396 & AT4G36540 & Bradi5g06620 & Pahal.G01160 & Sobic.001G105000 \\
\hline & & \multicolumn{8}{|c|}{ Salt tolerant-related protein } \\
\hline & & \multicolumn{8}{|c|}{ LEA hydroxyproline-rich glycoprotein family } \\
\hline & & \multicolumn{8}{|c|}{ Seed maturation protein PM41 } \\
\hline & & \multicolumn{8}{|c|}{ MADS-box transcription factor 1} \\
\hline & & \multicolumn{8}{|l|}{ Ethylene receptor } \\
\hline
\end{tabular}


grain growth by positively affecting LEN, kernel thickness, kernel width, and thousand kernel weight [17]. Overall, all the four genes control seed length or grain yield in rice, and the corresponding proteins are the putative candidate proteins of $L E N-3 H$ and $L E N-4 H$. Hence, the two major QTLs, $L E N-3 H$ and $L E N-4 H$, and the four putative candidate genes might play crucial and dynamic roles in the control of LEN in barley and other grain crops.

\section{Conclusion}

In this study, we identified two major QTLs for LEN (LEN-3H and $L E N-4 H$ ) derived from Baudin/Awcs276 and validated in Fleet/Awcs276. Additionally, four putative candidate genes that might control LEN and four categories of putative proteins that might have a phenotypic effect were identified for the two major QTLs. The QTLs and putative candidate genes identified in this study provide important information for barley genetic studies and breeding programs.

\section{Additional files}

Additional file 1: Phenotypic performance of barley kernel length of Baudin/Awcs276 recombinant inbred lines (RILs) population. (XLSX $11 \mathrm{~kb}$ )

Additional file 2: Analysis of variance (ANOVA) for kernel length of the Baudin/Awcs276 recombinant inbred line (RILs) population over the three years. (XLSX $10 \mathrm{~kb}$ )

Additional file 3: Average kernel length of the Baudin/Awcs276 recombinant inbred line (RILs) population. (XLSX 14 kb)

Additional file 4: Frequency distributions of kernel length in the Baudin/Awcs276 recombinant inbred line (RILs) population over the three years. (XLSX $26 \mathrm{~kb}$ )

Additional file 5: Genotyping information of the Baudin/Awcs276 recombinant inbred line (RILs) population. (XLSX 1007 kb)

Additional file 6: Linkage maps constructed using the Baudin/Awcs276 recombinant inbred line (RILs) population. (XLSX $60 \mathrm{~kb}$ )

Additional file 7: Information for high-resolution melt (HRM) markers developed based on the linkage genome-wide diversity array technology (DArT) markers. (XLSX $10 \mathrm{~kb})$

Additional file 8: Average kernel length of the Fleet/Awcs276 recombinant inbred line (RILs) population. (XLSX $11 \mathrm{~kb}$ )

Additional file 9: Genotyping information of the Fleet/Awcs276 recombinant inbred line (RILs) population. (XLSX $12 \mathrm{~kb}$ )

Additional file 10: Information regarding the peak marker within the interval of LEN-3H detected in 2015.. (XLSX 14 kb)

Additional file 11: Genotyping of the Baudin/Awcs 276 recombinant inbred line (RILs) population using the markers ABG453 and HVM40. (XLSX $12 \mathrm{~kb}$ )

Additional file 12: Mapping positions of the markers $A B G 453$ and HVM40 on linkage group (LG) 4 and LG6. (XLSX 20 kb)

\section{Abbreviations}

ANOVA: Analysis of variance; BLUP: Best linear unbiased prediction; Chr: Chromosome; cM: Centimorgan; DArT: Genome-wide diversity array technology; HRM: High-resolution melt; IM: Interval mapping; LEN: 10-Kerne length; LG: Linkage group; MLM: Mixed linear model; QTL: Quantitative trait locus; RIL: Recombinant inbred line; SNP: Single nucleotide polymorphism; SSR: Single sequence repeat

\section{Acknowledgements}

Not applicable.

\section{Funding}

This study was supported by the International Science and Technology Cooperation Program of China (No. 2015DFA30600) and the National Natural Science Foundation of China (31301317\& 31560388).

\section{Availability of data and materials}

All data generated or analyzed during this study are included in this published article and its supplementary information files.

\section{Authors' contributions}

$\mathrm{HZ}$ conducted data analysis and drafted the manuscript. SL helped to construct the research populations and performed the phenotypic evaluation. YL performed the phenotypic evaluation and helped to analyze the data. YL designed and coordinated this study and revised the manuscript. JY, MD, and GC participated in the construction of RIL population and phenotypic evaluation. JM developed the markers. YW participated in the design of the study. CL helped to draft the manuscript. YZ coordinated the study and helped to draft the manuscript. All authors have read and approved the final manuscript.

\section{Competing interests}

The authors declare that they have no competing interests.

\section{Consent for publication}

Not applicable.

Ethics approval and consent to participate Not applicable.

\section{Author details}

${ }^{1}$ Triticeae Research Institute, Sichuan Agricultural University, Wenjiang, Chengdu 611130, China. ${ }^{2}$ CSIRO Agriculture Flagship, 306 Carmody Road, St. Lucia, QLD 4067, Australia.

Received: 8 January 2016 Accepted: 6 September 2016

Published online: 13 September 2016

\section{References}

1. Moore G, Devos KM, Wang Z, et al. Cereal genome evolution: grasses, line up and form a circle. Curr Biol. 1995;5(7):737-9.

2. Graner A, Jahoor A, Schondelmaier J, et al. Construction of an RFLP map of barley. Theor Appl Genet. 1991;83(2):250-6.

3. Wenzl P, Carling J, Kudrna D, et al. Diversity Arrays Technology (DArT) for whole-genome profiling of barley. Proc Natl Acad Sci U S A. 2004;101(26): 9915-20.

4. Xue DW, Zhou MX, Zhang XQ, et al. Identification of QTLs for yield and yield components of barley under different growth conditions. J Zhejiang Univ Sci B. 2010;11(3):169-76.

5. Arifuzzaman M, Sayed MA, Muzammil S, et al. Detection and validation of novel QTL for shoot and root traits in barley (Hordeum vulgare L.). Mol Breedi. 2014;34(3):1373-87.

6. Wang J, Yang J, Jia Q, et al. A new QTL for plant height in barley (Hordeum vulgare L.) showing no negative effects on grain yield. PLoS One. 2014;9(2):e90144.

7. Ayoub M, Symons S, Edney M, et al. QTLs affecting kernel size and shape in a two-rowed by six-rowed barley cross. Theor Appl Genet. 2002;105(2-3): 237-47.

8. Backes G, Graner A, Foroughi-Wehr B, et al. Localization of quantitative trait loci (QTL) for agronomic important characters by the use of a RFLP map in barley (Hordeum vulgare L.). Theor Appl Genet. 1995;90(2):294-302.

9. Walker CK, Ford R, Muñoz-Amatriaín M, et al. The detection of QTLs in barley associated with endosperm hardness, grain density, grain size and malting quality using rapid phenotyping tools. Theor Appl Genet. 2013; 126(10):2533-51.

10. Han Y, Li D, Zhu D, et al. QTL analysis of soybean seed weight across multigenetic backgrounds and environments. Theor Appl Genet. 2012;125(4): 671-83

11. Wei L, Bai S, Li J, et al. QTL positioning of thousand wheat grain weight in Qaidam Basin. Open J Genet. 2014;4:239-44. 
12. Fan C, Xing Y, Mao H, et al. GS3, a major QTL for grain length and weight and minor QTL for grain width and thickness in rice, encodes a putative transmembrane protein. Theor Appl Genet. 2006;112(6):1164-71.

13. Wang $Y$, Xiong $\mathrm{G}, \mathrm{Hu}$ J, et al. Copy number variation at the GL7 locus contributes to grain size diversity in rice. Nat Genet. 2015;47(8):944-8.

14. Weng J, Gu S, Wan X, et al. Isolation and initial characterization of GW5, a major QTL associated with rice grain width and weight. Cell Res. 2008;18(12):1199-209.

15. Ishimaru K, Hirotsu N, Madoka Y, et al. Loss of function of the IAA-glucose hydrolase gene TGW6 enhances rice grain weight and increases yield. Nat Genet. 2013:45(6):707-11.

16. Luo J, Liu H, Zhou T, et al. An-1 encodes a basic helix-loop-helix protein that regulates awn development, grain size, and grain number in rice. Plant Cell. 2013;25(9):3360-76.

17. Xu F, Fang J, Ou S, et al. Variations in CYP78A13 coding region influence grain size and yield in rice. Plant Cell Environ. 2015;38(4):800-11.

18. Wang $\mathrm{H}$, Makeen $\mathrm{K}$, Yan $\mathrm{Y}$, et al. OsSIZ1 regulates the vegetative growth and reproductive development in rice. Plant Mol Biol Report. 2011;29(2): $411-7$.

19. Huang XY, Chao DY, Gao JP, et al. A previously unknown zinc finger protein, DST, regulates drought and salt tolerance in rice via stomatal aperture control. Genes Dev. 2009:23(15):1805-17.

20. Chen G, Liu Y, Ma J, Zheng Z, Wei Y, et al. A novel and major quantitative trait locus for fusarium crown Rot resistance in a genotype of wild barley (hordeum spontaneum L.). PLoS One. 2013;8(3):e58040. doi:10.1371/journal. pone.0058040

21. Smith SE, Kuehl RO, Ray IM, et al. Evaluation of simple methods for estimating broad-sense heritability in stands of randomly planted genotypes. Crop Sci. 1998;38(5):1125-9.

22. Allen GC, Flores-Vergara MA, Krasynanski S, et al. A modified protocol for rapid DNA isolation from plant tissues using cetyltrimethylammonium bromide. Nat Protoc. 2006;1(5):2320-5.

23. Meng L, Li H, Zhang L, et al. QTL IciMapping: Integrated software for genetic linkage map construction and quantitative trait locus mapping in biparental populations. Crop J. 2015;3(3):269-83.

24. Van Ooijen JW. Accuracy of mapping quantitative trait loci in autogamous species. Theor Appl Genet. 1992;84(7-8):803-11.

25. Van Ooijen JW. MapQTL version 6.0, Software for the mapping of quantitative trait loci in experimental populations of diploid species. Kyazma B. V., Wageningen, Netherlands. 2009.

26. Liu Y, Wang L, Sun C, et al. Genetic analysis and major QTL detection for maize kernel size and weight in multi-environments. Theor Appl Genet. 2014;127(5):1019-37.

27. Yang J, Hu CC, Hu H, et al. QTLNetwork: mapping and visualizing genetic architecture of complex traits in experimental populations. Bioinformatics. 2008;24(5):721-3.

28. Han Y, Khu DM, Monteros MJ. High-resolution melting analysis for SNP genotyping and mapping in tetraploid alfalfa (Medicago sativa L.). Mol Breed. 2012;29(2):489-501.

29. Zhang XY, Gao YN. To design PCR primers with Oligo 6 and primer premier 5 . China J Bioinform. 2004;2:14-8.

30. Nevo E, Chen GX. Drought and salt tolerances in wild relatives for wheat and barleyimprovement. Plant Cell Environ. 2010;33:670-85.

31. Wu D, Cai S, Chen M, Ye L, Chen Z, Zhang H, Dai F, Wu F, Zhang G. Tissuemetabolic responses to salt stress in wild and cultivated barley. PLoS One. 2013;8(1):e55431. 10.137 I/journal.pone.0055431.

32. Doebley JF, Gaut BS, Smith BD. The molecular genetics of crop domestication. Cell. 2006;127(7):1309-21.

33. Xing Y, Zhang Q. Genetic and molecular bases of rice yield. Annu Rev Plant Biol. 2010;61:421-42

34. Li S, Zhao B, Yuan D, et al. Rice zinc finger protein DST enhances grain production through controlling Gn1a/OsCKX2 expression. Proc Natl Acad Sci U S A. 2013;110(8):3167-72.

35. Wang H, Sun R, Cao Y, et al. OsSIZ1, a SUMO E3 ligase gene, is involved in the regulation of the responses to phosphate and nitrogen in rice. Plant and Cell Physiology. 2015;56(12):2381-95.

\section{Submit your next manuscript to BioMed Central and we will help you at every step:}

- We accept pre-submission inquiries

- Our selector tool helps you to find the most relevant journal

- We provide round the clock customer support

- Convenient online submission

- Thorough peer review

- Inclusion in PubMed and all major indexing services

- Maximum visibility for your research

Submit your manuscript at www.biomedcentral.com/submit
Biomed Central 